\title{
HEREDITARY PROPERTIES AND MAXIMALITY CONDITIONS WITH RESPECT TO ESSENTIAL EXTENSIONS OF LATTICE GROUP ORDERS
}

\author{
BY \\ JORGE MARTINEZ
}

\begin{abstract}
An $l$-group will be denoted by the pair $(G, P)$, where $G$ is the group and $P$ is the positive cone. The cone $Q$ is an essential extension of $P$ if every convex $l$-subgroup of $(G, Q)$ is a convex $l$-subgroup of $(G, P) . Q$ is very essential over $P$ if it is essential over $P$ and for each $0 \neq x \in G$ and each $Q$-value $D$ of $x$, there is a unique $P$-value $C$ of $x$ containing $D$. We seek conditions which are preserved by essential extensions; normal valuedness and the existence of a finite basis are so preserved.

We then investigate $l$-groups which have the property that their positive cone has no proper very essential extensions. $Q$ is a c-essential extension of $P$ if $Q$ is essential over $P$ and every closed convex $l$-subgroup of $(G, Q)$ is closed in $(G, P)$. We show that a wreath product of totally ordered groups has no proper very $c$-essential extensions. We derive a sufficient condition for the nonexistence of such extensions in case the l-group has property (F): no positive element exceeds an infinite set of pairwise disjoint elements.
\end{abstract}

Introduction. The theory presented here grew out of an attempt to answer some of the questions raised in [7]. In that paper we defined the notions of essential and very essential extensions of lattice cones (henceforth: $l$-cones). In this work we investigate the following: we wish to know which properties of $l$-groups are preserved by essential extensions ( $\$ 2)$; then we look for conditions which imply that an $l$-cone has no proper very essential extensions (\$3).

It is shown in [7] that representability and finite valuedness of l-groups are both preserved by essential extensions. We prove in this paper that normal valuedness is preserved. With certain restrictions on the type of essential extensions, we can show that lateral completeness and local representability (defined in \$2) are preserved also. We give an example to show property $(F)$ and the existence of a basis are not preserved.

An essential extension $Q$ of $P$ is $c$-essential if every $Q$-closed convex $l$-subgroup of $(G, Q)$ is $P$-closed. In $\S 3$ we consider $l$-groups which have the following property: for each pair of disjoint special elements $a$ and $b$ there is an $x \in G$ such that $a^{x}=-x$ $+a+x$ and $b$ are comparable; for any such $x$ and $n=1,2, \ldots, a=a^{n x}$ or else $a \wedge a^{n x}$

Received by the editors October 27, 1970.

AMS 1970 subject classifications. Primary 06A55.

Key words and phrases. Essential extensions of lattice group orders, $c$-essential extensions, hereditary properties, property (S), property (F), locally representable l-groups, laterally complete 1-groups. 
$=0$. This condition will be called property (S). We show (Theorem 3.2) that if $(G, P)$ is a laterally complete $l$-group with the local representation property and property (S) then $P$ has no proper very $c$-essential extensions. This implies that if $H$ wr $K$ is the wreath product of totally ordered groups (abbr. o-groups) $H$ and $K$, then its cone has no proper very $c$-essential extensions. Theorem 3.4 says that the cone of an l-group with properties (F) and (S) has no proper very essential extensions.

1. Preliminaries and definitions. Throughout, let $(G, P)$ denote an $l$-group, where $G$ is the group and $P$ is the positive cone. In general we shall use the same notation and terminology of [7], notably: $\mathscr{C}(P)$ denotes the lattice of convex l-subgroups of $(G, P) . M \in \mathscr{C}(P)$ is regular if it is maximal in $\mathscr{C}(P)$ with respect to not containing some element $g \in G$. $\mathscr{R}(P)$ will denote the set of regular subgroups of $\mathscr{C}(P)$. It is well known that $\mathscr{R}(P)$ is a root system [4]; that is, no two incomparable regular subgroups have a common lower bound in $\mathscr{R}(P)$. A regular subgroup $M$ is a value of $x \in G$ if $M$ is maximal without $x$. If $\bar{M}=\bigcap\{C \in \mathscr{C}(P): C \supset M\}$ then $\bar{M} \supset M$ and $x \in \bar{M} \backslash M$; obviously $\bar{M}$ covers $M$. If $M$ is normal in $\bar{M}$ then $\bar{M} / M$ is an archimedean $o$-group and hence $o$-isomorphic to a subgroup of the real numbers. A subgroup $B \in \mathscr{C}(P)$ is prime if $a \wedge_{P} b=0$ implies that $a \in B$ or $b \in B$; a regular subgroup is always prime [4].

$(G, P)$ is finite valued if every nonzero element of $G$ has at most a finite number of values. This is equivalent to saying that each $M \in \mathscr{R}(P)$ is special [4]; that is, $M$ is the value of an element $y$ having only one value. (The element $y$ itself is called special.) It is proved in [4] that a nonzero element $g$ is finite valued if and only if $g$ can be written as a sum of special elements $g=g_{1}+\cdots+g_{n}$, such that $\left|g_{i}\right| \wedge_{P}\left|g_{j}\right|$ $=0$ for $i \neq j$.

An l-group $(G, P)$ is completely distributive if the most general distributive law relative to meet and join in $G$ holds. Byrd and Lloyd have shown in [1] that $(G, P)$ is completely distributive if and only if every nonzero element of $G$ has a closed value. $(C \in \mathscr{C}(P)$ is closed if $C$ is closed under all existing joins and meets.)

An $l$-cone $Q$ extends $P$ if $Q \supseteq P ; Q$ is an essential extension of $P$ if $\mathscr{C}(Q) \subseteq \mathscr{C}(P)$ (we also say $Q$ is essential over $P$ ). The following two results are crucial in the development of our theory.

1.1 LEMMA [7]. If $Q$ is essential over $P$ then for each $0 \neq x \in G$ and each $Q$-value $D$ of $x$, there is a $P$-value $C$ of $x$ that contains $D$ and no other $Q$-value of $x$.

1.2 TheOREM [7]. An extension $Q$ of $P$ is essential over $P$ if and only if each $x \in Q$ is archimedean equivalent to $x_{P}^{+}=x \vee_{P} 0$ relative to $Q$. (Two positive elements $a$ and $b$ are archimedean equivalent if there exist positive integers $m$ and $n$ such that $m a \geqq b$ and $n b \geqq a$; notation: $a \sim b$.) 
An essential extension $Q$ of $P$ is very essential over $P$ if for each $0 \neq x \in G$ and each $Q$-value $D$ of $x$ there is exactly one $P$-value $C$ of $x$ that contains $D$. This is equivalent to saying that for each $0 \neq x \in Q$ and each $Q$-value $D$ of $x$ we have $D+x=D+x_{P}^{+}[7$, Theorem 1.3].

Let $(G, P)$ be an $l$-group; for $a, b \in P$ we write $a \ll b$ if $n a<b$ for all $n=1,2, \ldots$ We say that $a$ is infinitely small relative to $b$. The $l$-group $(G, P)$ is normal valued if each regular subgroup $D$ of $G$ is normal in $\bar{D}$. We get the following corollary.

1.3.1 CoRollary [7]. Let $Q$ be a very essential extension of $P$. Then, for each $x \in Q \mid 0, x_{P}^{-}=(-x) \vee_{P} 0 \ll x_{P}^{+}$. Conversely, if $Q$ extends $P$ and $(G, Q)$ is normal valued and $x_{P}^{-} \ll x_{P}^{+}$for each $0 \neq x \in Q$ then $Q$ is very essential over $P$. (Both inequalities here are taken relative to $Q$.)

Finally if $S$ is any subset of the group $G,\langle S\rangle$ denotes the subgroup generated by $S$.

2. $c$-essential extensions and hereditary properties of $l$-cones. A property of an l-group is said to be hereditary if it is preserved by all essential extensions of the cone. A property $\Phi$ is universally hereditary if it is preserved by any essential extension of the $l$-cone of each $l$-group possessing property $\Phi$. It is shown in [7] that representability of an $l$-group is universally hereditary. (Recall that an $l$-group $(G, P)$ is said to be representable if it can be embedded as an $l$-subgroup of a cardinal product of $o$-groups.) Proposition 1.13 in [7] states that complete distributivity is universally hereditary; the proof given there is not valid however. That argument does show that if $(G, P)$ is normal valued then complete distributivity is hereditary. Our first result in this section shows that normal valuedness itself is universally hereditary. We shall use a result of Wolfenstein [8, Theorem 3] which says among other things that $(G, P)$ is normal valued if and only if $A+B=B+A$ for all $A, B \in \mathscr{C}(P)$.

2.1 Proposition. If $(G, P)$ is a normal valued l-group and $Q$ is an essential extension of $P$ then $(G, Q)$ is normal valued.

Proof. Let $A, B \in \mathscr{C}(Q)$; since $Q$ is essential over $P$ we have $\mathscr{C}(Q)$ contained in $\mathscr{C}(P)$, and so $A+B=B+A$ since $(G, P)$ is normal valued.

The next theorem is a generalization of Theorem 1.12 in [7] and gives a partial converse to the previous result.

2.2 THEOREM. Let $(G, P)$ be an l-group, $Q$ be a very essential extension of $P$ such that $(G, Q)$ is normal valued l-group. Then every nonzero element of $G$ has a normal $P$-value.

Proof. Let $D \in \mathscr{R}(Q)$ and $0<x \in \bar{D} \backslash D$ (rel. $P$ ). Let $C_{1}$ be the unique $P$-value of $x$ that contains $D$. Define a map $\eta: \mathscr{R}(Q) \rightarrow \mathscr{R}(P)$ by $C_{1}=D \eta$; we must show this map is well defined. So let $0<y \in \bar{D} \mid D$ (rel. $P$ ) and $C_{2}$ be the $P$-value of $y$ that contains $D$. First of all, $C_{1}$ and $C_{2}$ must be comparable (the proof of this fact is identical to the one in the proof of 1.12 in [7]). So suppose that $C_{1} \subset C_{2}$; since $D$ 
is normal in $\bar{D}$ we may assume (by taking a suitable multiple) that $D+x>D+y$. Thus, for some $0 \neq d \in D \cap P, d+x>y$ (rel. $Q$ ); notice that $C_{1}$ is still a $P$-value of $d+x$. The assumption that $C_{1} \subset C_{2}$ excludes that $d+x \geqq y$ (rel. $P$ ), and of course $d+x<y$ (rel. $P$ ) is impossible. Therefore, if $a=(d+x) \wedge_{P} y$ and $y^{\prime}=y-a$ and $x^{\prime}$ $=(d+x)-a$, then $0 \neq x^{\prime}, y^{\prime} \in P$ and $x^{\prime} \wedge_{P} y^{\prime}=0$. This implies that $x^{\prime} \wedge_{Q} y^{\prime} \ll x^{\prime} \vee_{Q} y^{\prime}$ (rel. $Q$ ) [7, Corollary 1.1.3]; hence $y \ll d+x$ (rel. $Q$ ). There are two cases to consider:

I. $a \in D$; then $D+y \ll D+d+x=D+x$ relative to $Q$ in $\bar{D} / D$, which is absurd.

II. $a \in \bar{D} \backslash D$; since $d+x \in C_{2}$ (because $C_{1} \subset C_{2}$ ), it follows that $a \in C_{2}$. But then $C_{2}$ is a $P$-value of $y^{\prime}=y-a$, so in particular $y-a \notin C_{2}$ and hence not in $D$. Thus $D \neq D+y^{\prime} \ll D+x^{\prime}$ (rel. $Q$ ), once again a contradiction.

Conclusion. $C_{1} \notin C_{2}$, and by symmetry $C_{2} \notin C_{1}$; hence $C_{1}=C_{2}$. This establishes that the map $\eta$ is indeed well defined.

Next, let $C=D \eta$; then $C \cap \bar{D}=D$ by the definition of $\eta$, because $0<x \in \bar{D} \mid D$ (rel. $P$ ) implies that $x \in \bar{C} \mid C$. We also get that $\bar{D} \subseteq \bar{C}$. Now $\langle\bar{D} \cup C\rangle$ is a convex l-subgroup of $(G, P)$ contained in $\bar{C}$, and it contains $C$ properly; therefore $\bar{C}=$ $\langle\bar{D} \cup C\rangle$. Then, as in the proof of 1.12 in [7] one shows that $C+d=d+C$ for all $d \in \bar{D}$, proving that $\bar{D}+C=C+\bar{D}=\langle\bar{D} \cup C\rangle=\bar{C}$ and finally that $C$ is normal in $\bar{C}$. Moreover,

$$
\bar{C} / C=\bar{D}+C / C \cong \bar{D} / \bar{D} \cap C=\bar{D} / D \quad \text { (with order) }
$$

This proves the theorem since every nonzero element of $G$ has a $P$-value in the image of $\mathscr{R}(Q)$ under $\eta$.

Remark. The map $\eta$ defined above is $1-1$ and $\eta^{-1}$ preserves order. We show two things:

(1) With $D_{1}, D_{2} \in \mathscr{R}(Q)$ and $D_{1} \| D_{2}$ it follows that $D_{1} \eta \| D_{2} \eta$. For we can pick $c, d \in Q \mid 0$ such that $c \wedge_{Q} d=0, c \in D_{1}\left|D_{2}, d \in D_{2}\right| D_{1}$ and also $c \in \bar{D}_{2}$ while $d \in \bar{D}_{1}$. Take $a=c_{P}^{+}$and $b \in d_{P}^{+}$; then relative to $Q, a \sim c$ and $b \sim d$, so that $D_{1}$ (resp. $D_{2}$ ) is a $Q$-value of $b$ (resp. $a$ ). In addition $a \wedge_{P} b=0$, consequently $D_{1} \eta \| D_{2} \eta$, since $D_{1} \eta$ and $D_{2} \eta$ are $P$-values of $b$ and $a$ respectively.

(2) Next, if $D$ and $D^{\prime}$ are in $\mathscr{R}(Q)$ and $D \eta \subseteq D^{\prime} \eta$, we prove that $D \subseteq D^{\prime}$. By (1), $D$ and $D^{\prime}$ must be comparable, so suppose $D^{\prime} \subset D$. Then $\bar{D}^{\prime} \subseteq D \subseteq D \eta \subseteq D^{\prime} \eta$ and using the fact that $\bar{D}^{\prime} \cap D^{\prime} \eta=D^{\prime}$ we get that $D^{\prime}=\bar{D}^{\prime}$, which is absurd.

In particular, if $D \eta=D^{\prime} \eta$ it follows that $D=D^{\prime}$, proving $\eta$ is 1-1.

Recall that an $l$-group $(G, P)$ is representable if and only if $a \in P$ and $a \wedge_{P} a^{x}=0$ for some $x \in G$ imply that $a=0$ [6, Theorem 1.8]. (Of course, $a^{x}=-x+a+x !$ ) As an aside on Theorem 2.2 we can give the following characterization of representability, which improves on Proposition 1.11 in [7].

2.3 THEOREM. The following statements about the l-group $(G, P)$ are equivalent:

(i) $(G, P)$ is representable;

(ii) $P$ is the meet of total very essential extensions;

(iii) $P$ is the meet of total orders;

(iv) $P$ is the meet of $l$-cones $Q_{\lambda}$ such that each $\left(G, Q_{\lambda}\right)$ is representable. 
Proof. It is proved in Theorem 1.5 of [7] that (i) implies (ii); that (ii) implies (iii) is trivial. (iii) obviously implies (iv) and since (i) implies (iii), (iv) also implies (iii). Finally, suppose (iii) holds; take $a \in P$ and assume $a \wedge_{P} a^{x}=0$ for some $x \in G$. There is a total order $Q_{1}$ (resp. $Q_{2}$ ) which extends $P$ and such that $a<a^{x}$ relative to $Q_{1}$ (resp. $a^{x}<a$ relative to $Q_{2}$ ). Thence

$$
\begin{array}{lll}
a<a^{x}<a^{2 x}<\cdots<a^{n x}<\cdots & \left(\text { rel. } Q_{1}\right), \\
a>a^{x}>a^{2 x}>\cdots>a^{n x}>\cdots & \text { (rel. } \left.Q_{2}\right) .
\end{array}
$$

Thus if we take any total order $Q$ which extends $P$ then one of the above string of inequalities must hold.

Consider the element $b=a-a^{x}+a^{2 x} ; b \in Q$ for each total order $Q$ extending $P$. On the other hand, $b=-a^{x}+\left(a+a^{2 x}\right)$, since $a \wedge_{P} a^{x}=0$; also $a^{2 x} \wedge_{P} a^{x}=0$ and so $a^{x} \wedge_{P}\left(a+a^{2 x}\right)=0$. Therefore $b \notin P$; this is a contradiction unless $a=0$. We conclude then that $(G, P)$ is representable, and the theorem has been proved.

An essential extension $Q$ of $P$ is called c-essential if every closed convex $l$-subgroup of $(G, Q)$ is also closed in $(G, P) . Q$ is very c-essential over $P$ if $Q$ is a very essential extension of $P$ which is also $c$-essential over $P$. (We will consider some implications of this restriction at the end of this section.) A property $\Phi$ of an l-group $(G, P)$ is $c$-hereditary if each $c$-essential extension of $P$ has property $\Phi$. The property $\Phi$ is universally c-hereditary if it is preserved by any $c$-essential extension of each $l$-group having property $\Phi$.

An l-group $(G, P)$ is said to satisfy the local representation property if each nonzero element $g$ in $P$ can be written as the join of special elements which are pairwise disjoint. Conrad has shown this is equivalent to saying that the subset $\mathscr{S}(P)$ of special regular subgroups is plenary [5]. (A subset $\mathscr{R} \subseteq \mathscr{R}(P)$ is plenary if (1) $\bigcap \mathscr{R}=0$ and (2) $\mathscr{R}$ is a dual ideal; that is, if $M \in \mathscr{R}$ and $M \subseteq D \in \mathscr{R}(P)$ then $D \in \mathscr{R}$.)

2.4 Proposition. The local representation property is universally c-hereditary.

Proof. Let $(G, P)$ be an $l$-group with the local representation property, and $Q$ be a $c$-essential extension of $P$. The set $\mathscr{P}(P)$ of special regular subgroups is plenary, and hence [8, Theorem 3] $(G, P)$ is normal valued. Also each special regular subgroup is essential and therefore closed [2, Proposition 4.1]; so $(G, P)$ is completely distributive. Consequently, $(G, Q)$ is completely distributive since this property is hereditary for normal valued $l$-groups. According to Theorem 3.4 of [1] $\mathscr{R}(Q)$ has a plenary subset consisting of $Q$-closed subgroups. Pick any closed, regular subgroup of $(G, Q)$, say $D$; it is $P$-closed by our assumption. Now, suppose $D$ is a value of $a \in P$, and write $a=\bigvee_{P} a_{i}(i \in I)$ as a join of pairwise $P$-disjoint, $P$-special elements. Certainly each $a_{i} \in \bar{D}$ by convexity and since $D$ is $P$-closed some $a_{j} \notin D$; therefore $D$ is $Q$-special.

The above argument shows every closed, regular subgroup of $(G, Q)$ is special, and so $\mathscr{S}(Q)$ is a plenary subset of $\mathscr{R}(Q)$. This proves that $(G, Q)$ has the local representation property. 
An l-group $(G, P)$ is laterally complete if every set of pairwise disjoint elements has a least upper bound.

2.5 TheOREM. If $(G, P)$ is laterally complete and $Q$ is a c-essential extension of $P$ such that $(G, Q)$ is completely distributive, then $(G, Q)$ is also laterally complete.

Proof. Let $\left\{a_{i}: i \in I\right\}$ be a family of $Q$-positive elements which are pairwise $Q$-disjoint. For each $i \in I$, let $b_{i}=\left(a_{i}\right)_{P}^{+}$and $c_{i}=\left(a_{i}\right)_{P}^{-}$. Since $Q$ is essential over $P$, $b_{i} \sim a_{i}$ (rel. $Q$ ), for all $i \in I$, and so $b_{i} \wedge_{Q} b_{j}=0$ for $i \neq j$. This implies that the $b_{i}$ are mutually $P$-disjoint, and hence $b=\bigvee_{P} b_{i}$ exists. Since $b_{i} \geqq c_{i}$ (rel. $Q$ ), for all $i \in I$, it follows that the $c_{i}$ are also pairwise $Q$-disjoint and therefore $P$-disjoint; again $c=\bigvee_{P} c_{i}$ exists.

We claim $b=\bigvee_{Q} b_{i}$ and $c=\bigvee_{Q} c_{i}$. Clearly $b \geqq b_{i}$ (rel. $Q$ ) for each $i \in I$; take $y \geqq b_{i}$ (rel. $Q$ ) for all $i \in I$. $(G, Q)$ is completely distributive, so pick a $Q$-closed $Q$-value of $b-y$, say $M$. If $b \in M$ then $M+b-y=M-y<M$; if $b \notin M$ then since $M$ is also $P$-closed, some $b_{j} \notin M$. If $i \neq j$ then $b_{i} \in M$ because $M$ is $Q$-prime and $b_{i}$ is disjoint to $b_{j}$. Therefore $M+b-y=M+b_{j}-y<M$. Consequently, $y \geqq b$ (rel. $Q$ ) proving $b=\bigvee_{Q} b_{i}$; similarly $c=\bigvee_{Q} c_{i}$.

Finally we show $b-c=\bigvee_{Q} a_{i}$; for $b_{i} \geqq c_{i}$ (rel. $Q$ ) which immediately implies that $b-c \in Q$. Clearly each $b_{i}$ commutes with any $b_{j}$; the same thing is true of the $c_{i}$. Also each $b_{i}$ commutes with any $c_{j}$. Thus,

$$
(b-c)-a_{i}=\left(b-b_{i}\right)-\left(c-c_{i}\right)=\bigvee_{Q} b_{j \neq i}-\bigvee_{Q} c_{j \neq i}
$$

where the joins are taken as indicated over all $j \neq i$. The last term is of course $Q$-positive and hence $b-c \geqq a_{i}$ (rel. $\left.Q\right)$, for each $i \in I$. If $y \geqq a_{i}(i \in I)$, then $y+c_{i}$ $\geqq b_{i}(i \in I)$; now $\bigvee_{Q}\left(y+c_{i}\right)$ exists and is $y+\left(\bigvee_{Q} c_{i}\right)=y+c$. Hence $y+c \geqq b$, that is, $y \geqq b-c$, finally proving $b-c=\bigvee_{Q} a_{i}$.

2.5.1 Corollary. Let $(G, P)$ be a normal valued l-group which is completely distributive. Then lateral completeness is c-hereditary.

Proof. Since $(G, P)$ is normal valued, complete distributivity is hereditary.

The following remark shows that at least in the context of finite valued $l$-groups there is no difference between essential and $c$-essential extensions.

2.6 Proposition. Let $(G, P)$ be a finite valued l-group. Every convex l-subgroup of $(G, P)$ is closed.

Proof. First, every regular subgroup is special and hence closed. But every convex $l$-subgroup is the meet of regular subgroups, all of which are closed. Therefore every convex $l$-subgroup must be closed.

2.6.1 Corollary. If $(G, P)$ is a finite valued l-group, then every essential extension of $P$ is c-essential. 
An element $x>0$ in an $l$-group $(G, P)$ is basic if $\{g \in G: 0 \leqq g \leqq x\}$ is a chain. A basis for $(G, P)$ is a maximum set of pairwise disjoint elements each of which is basic. An l-group has a finite basis if and only if every set of pairwise disjoint elements is finite [3, Corollary I to Theorem 5.2]. We point out that an $l$-group has a finite basis if and only if it is finite valued and laterally complete. (Necessity is obvious, and if $(G, P)$ is both finite valued and laterally complete, then a set of pairwise disjoint elements cannot have more than a finite number of elements.) We therefore get

\subsubsection{COROLLARY. The property of having a finite basis is universally hereditary.}

Proof. Finite valuedness is universally hereditary, and in the context of finite valued $l$-groups (which are completely distributive) lateral completeness is also hereditary in view of Theorem 2.5 and Corollary 2.6.1.

We now give an example of properties that are not preserved by essential extensions. An l-group $(G, P)$ has property $(\mathrm{F})$ if no positive element of $G$ exceeds an infinite set of pairwise disjoint elements. An l-group with property $(\mathrm{F})$ has a basis [3, Theorem 5.2].

Let $(G, P)=\boldsymbol{R} \boxplus \boldsymbol{R} \boxplus \boldsymbol{R} \ldots$; that is, $G$ is the restricted sum of countably many copies of the reals and $P$ is the pointwise ordering on $G$. It is evident that $(G, P)$ has property (F). Let $Q$ be the lattice order induced on $G$ by the following order of the natural numbers. In other words, $x \in Q$ if and only if its maximal nonzero

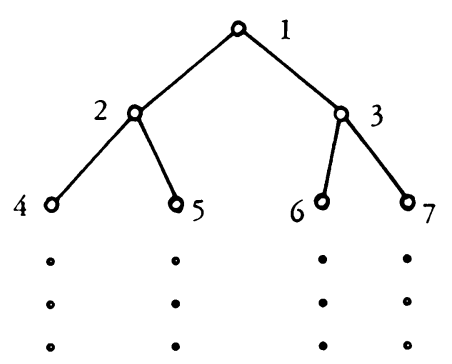

components relative to the above order are positive. It is clear that $(G, Q)$ has no basic elements and from Proposition 2.5 in [7] it follows that $Q$ is a very essential extension of $P$. But since $(G, Q)$ has no basis it cannot satisfy property (F). Thus property $(\mathrm{F})$ is not hereditary for $(G, P)$; neither is the property of having a basis. Notice also that since $(G, P)$ is finite valued, these properties are not $c$-hereditary either.

Let $(G, P)$ be an $l$-group satisfying the local representation property. Let $Q$ be a very $c$-essential extension of $P$ and let $\eta$ be the map defined in the proof of 2.2 (it makes sense to define $\eta$ because $(G, Q)$ has the local representation property, according to 2.4 , and is therefore normal valued). In this situation the restriction of $\eta$ to $\mathscr{S}(Q)$ is onto $\mathscr{S}(P)$. For if $C \in \mathscr{S}(P)$, pick a $P$-special element $u \in \bar{C} \mid C$; $u$ is $Q$-special and its $Q$-value $D$ is contained in $C$. By the definition of $\eta$ it is obvious that $C=D \eta$; of course $D \in \mathscr{S}(Q)$. Next, if $M \in \mathscr{S}(Q)$, there is an element $x$ whose 
only $Q$-value is $M$; we may assume $x \in P$ since $Q$ is very essential over $P$ (see also Corollary 1.3.1 in [7]). Write $x=\bigvee_{P} x_{i}$ as a join of $P$-disjoint, $P$-special elements. $M$ is $P$-closed which implies that $M$ is the $P$-value of some $x_{j}$. Again using the definition of $\eta$ we obtain $x_{j} \in(M \eta)^{-} \mid M \eta$, proving $M \eta \in \mathscr{C}(P)$.

The arguments above prove the following theorem:

2.7 THEOREM. Let $(G, P)$ be an l-group satisfying the local representation property; if $Q$ is a very c-essential extension of $P$, the map $\eta: \mathscr{S}(Q) \rightarrow \mathscr{S}(P)$, defined by $C=D \eta$ if and only if $C$ is the $P$-value of $g$ containing $D$ whenever $D$ is a $Q$-value of $g$, is a 1-1 map onto $\mathscr{S}(P)$ such that $\eta^{-1}$ preserves order. If $C=D \eta$ then $\bar{C} / C$ is $o$-isomorphic to $\bar{D} / D$. Finally, $C=D \eta$ if and only if $D$ is the $Q$-value of $x$, whenever $x$ is an element whose only $P$-value is $C$.

2.7.1 COROLlaRY. Let $(G, P)$ be a finite valued l-group, and $Q$ be a very essential extension of $P$. The map $\eta: \mathscr{R}(Q) \rightarrow \mathscr{R}(P)$ defined as in 2.7 is a 1-1 map onto $\mathscr{R}(P)$ such that $\eta^{-1}$ preserves order. If $C=D \eta$ then $\bar{C} / C$ and $\bar{D} / D$ are isomorphic as o-groups.

NoTE. This corollary was proved in [7] for very essential extensions of certain finite valued abelian p.o. groups.

To close this section let us show that any abelian $l$-group having an $l$-ideal which is not closed has an essential extension to a total order which is not $c$-essential. So suppose $(G, P)$ is an abelian $l$-group and $K$ is an $l$-ideal that is not closed. Let $P_{K}$ be the cone induced in $G / K$ by $P$. Take $Q^{\prime}$ to be an essential extension of $K \cap P$ in $K$, and $Q^{\prime \prime}$ an essential extension of $P_{K}$ in $G / K$. We may choose both of these to be total orders in view of Theorem 1.5 in [7]. Define $Q$ on $G$ as follows: Let $x$ be $Q$-positive if $x \in Q^{\prime}$ or else $x \notin K$ and $K+x \in Q^{\prime \prime}$. It is clear that $Q$ is a total order on $G$; in fact $(G, Q)$ is a lexicographic extension of $\left(K, Q^{\prime}\right)$ by $\left(G / K, Q^{\prime \prime}\right)$. Thus if $x \in Q \backslash K$ and $y \in K$ then $x>y$ (rel. $Q$ ). This implies that $K$ is a $Q$-closed convex subgroup. For if $\left\{x_{\alpha}: \alpha \in A\right\} \subseteq K \cap Q$ and $x=1$.u.b. $\left\{x_{\alpha}\right\}$ exists, then $x \in K$. Otherwise, let $0<y \in K$ (note: since $K$ is not $P$-closed, $K \neq 0$ ). Then $x>y>0$ (rel. $Q$ ), and $x-y \notin K$. But then $x>x-y>x_{\alpha}$, for each $\alpha$, contradicting the definition of $x$.

$Q$ clearly extends $P$, and if $g \in Q$ with $g \in K$, then $g \sim g_{P}^{+}$(rel. $Q^{\prime}$ ), and hence relative to $Q$, since $Q^{\prime}$ is essential over $K \cap P$. If $g \in K$ then $g_{P}^{+} \notin K$; otherwise $g_{P}$ is also in $K$, because $g_{P}^{+}>g_{P}^{-}$(rel. $Q$ ). Thus $K+g \sim K+g_{P}^{+}=(K+g)_{P}^{+}$(rel. $Q^{\prime \prime}$ ), proving $g \sim g_{P}^{+}$(rel. $Q$ ) and hence $Q$ is essential over $P$. But by the previous paragraph $Q$ is not $c$-essential over $P$.

RemarK. In the above discussion $K$ will be $Q$-regular if it is $P$-regular. It is shown in [4, Theorem 3.1] that a convex $l$-subgroup is regular if and only if it is meet irreducible in the lattice $\mathscr{C}^{\prime}(P)$. (If $L$ is a lattice then $a \in L$ is meet irreducible if $\bigwedge\left\{b_{i}: i \in I\right\}=a$ implies that some $b_{i}=a$.) Using this fact about regular subgroups one easily shows that if $K$ is $P$-regular it is also $Q$-regular.

Notice that this fact does not depend upon the order $Q$ constructed in the preceding discussion or upon commutativity of $G$ for that matter. In fact, if $(G, P)$ 
is any $l$-group and $Q$ is an essential extension of $P$, then if $K$ is a $P$-regular subgroup of $(G, P)$ which happens to be in $\mathscr{C}(Q)$, we can conclude that $K$ is $Q$-regular as well.

3. Maximality of orders relative to $c$-essential extensions. In this section we investigate the question of maximality of $l$-cones with respect to certain essential extensions. Before going on with the discussion we record the following lemma:

3.1 Lemma. Let $(G, P)$ be an l-group with the local representation property. If $Q$ is a c-essential extension of $P$ having the property that $a \wedge_{P} b=0$ implies $a \wedge_{Q} b=0$, for any two $P$-special elements $a$ and $b$, then $P=Q$.

Proof. Let $a \in P$, and write $a=\bigvee_{P} a_{i}$ as a join of pairwise $P$-disjoint, $P$-special elements. As in the proof of 2.5 one shows $a=\bigvee_{Q} a_{i}$.

Now let $x \in Q$ and consider $x_{P}^{+}$and $x_{P}^{-}$. If $x_{P}^{-} \neq 0$ then, writing both elements as joins in $P$ of pairwise $P$-disjoint, $P$-special components, say $x_{P}^{+}=\bigvee_{P} c_{i}$ and $x_{P}^{-}$ $=\bigvee_{P} d_{\alpha}$, we can conclude that each $c_{i}$ is $P$-disjoint from each $d_{\alpha}$. But then $c_{i} \wedge_{Q} d_{\alpha}=0$, for each $i$ and each $\alpha$ by our assumption; this clearly implies, from the remark in the previous paragraph, that $x_{P}^{+} \Lambda_{Q} x_{P}^{-}=0$, which is absurd. We must therefore conclude that $x_{P}^{-}=0$ and $Q=P$.

In the ensuing discussion $(G, P)$ will be a laterally complete l-group with the local representation property, and in addition we will assume $(G, P)$ satisfies the following: for any two disjoint, special elements $a$ and $b$ there is an $x \in G$ such that $a^{x}=-x+a+x$ and $b$ are comparable. For any such $x$ and $n=1,2, \ldots, a \wedge a^{n x}=0$ or $a=a^{n x}$. The condition just described will be called property (S).

Let us give an example of a class of $l$-groups satisfying all of the above requirements. For any two $o$-groups $H$ and $K$, let $(G, P)$ be the unrestricted wreath product of $H$ with $K$, denoted by $H$ wr $K$. In other words, $G=H \times \prod\left\{K_{h}: h \in H\right\}$ where each $K_{h}=K$; the operation on $G$ is defined by

$$
\left(f ; \ldots, a_{h}, \ldots\right)+\left(g ; \ldots, b_{h}, \ldots\right)=\left(f+g ; \ldots,\left(a_{h-g}+b_{h}\right)_{h}, \ldots\right),
$$

for all $f, g \in H$, and, for each $h \in H, a_{h}, b_{h} \in K$. An element of the group is in $P$ if and only if $f>0$ in $H$ or $f=0$ and each $a_{h} \geqq 0$ in $K$. $H$ wr $K$ is obviously a laterally complete $l$-group with the local representation property. If $a$ and $b$ are disjoint, special elements of $H$ wr $K$ then $a=\left(0 ; \ldots, 0, c_{h}, 0, \ldots\right)$ and $b=\left(0 ; \ldots, 0, d_{l}, 0\right.$, $\ldots)$ with $h \neq l$. Let $x=(-h+l ; \ldots, 0,0,0, \ldots)$; then $a^{x}=\left(0 ; \ldots, 0,\left(c_{h}\right)_{l}, 0, \ldots\right)$ which is disjoint from $a$ but comparable to $b$. It is easy to check that $a \wedge a^{n x}=0$ for all $n=1,2, \ldots$; it follows therefore that $H$ wr $K$ has property (S).

Another example of an $l$-group with these properties is the following: let $(G, P)$ $=\boldsymbol{Z} \times \boldsymbol{Z} \times \boldsymbol{Z}$ where $\boldsymbol{P}=\{(a, b, c): c>0$ in $\boldsymbol{Z}$, or $c=0$ and $a, b \geqq 0\}$ and the addition is defined by

$$
\begin{aligned}
(a, b, c)+(x, y, z) & =(a+x, b+y, c+z) \quad \text { if } z \text { is even, } \\
& =(b+x, a+y, c+z) \quad \text { if } z \text { is odd }
\end{aligned}
$$


for all $a, b, c, x, y, z \in Z$. It is immediate that $(G, P)$ is laterally complete and has the local representation property. Two elements $g$ and $h$ are disjoint if and only if $g=(a, 0,0)$ and $h=(0, b, 0)$ or vice versa, for some positive integers $a$ and $b$. Let $t=(0,0,1)$; then $g^{t}=(0, a, 0)$, so $g$ is disjoint from $g^{t}$ and $h$ is comparable to $g^{t}$. Further $g^{t}=g^{3 t}=g^{5 t}=\cdots$ and $g^{2 t}=g^{4 t}=\cdots=g$ and once again $(G, P)$ has property (S).

Once more let $(G, P)$ be a laterally complete $l$-group with the local representation property and also property (S). We will show $P$ has no proper very $c$-essential extensions. Suppose by way of contradiction that $Q$ is a proper very $c$-essential extension of $P$; by Lemma 3.1 there exist $P$-disjoint, $P$-special elements $a$ and $b$ which are no longer $Q$-disjoint. But then by Theorem 1.4 in [7] we may assume $a \ll b$ (rel. $Q$ ).

Case I. There exists an $x \in G$ such that $b \leqq a^{x}$ (rel. $P$ ); then $a \ll a^{x}$ (rel. $Q$ ) and hence

$$
a \ll a^{x} \ll a^{2 x} \ll \cdots \ll a^{n x} \ll \cdots \quad(\text { rel. } Q) .
$$

But $a$ is then $P$-disjoint to all the $a^{n x}$ and consequently the $a^{n x}$ are pairwise $P$-disjoint. Therefore, since $(G, P)$ is laterally complete $g=a \vee_{P} a^{x} \vee_{P} a^{2 x} \vee_{P} \cdots$ exists; $(G, Q)$ is certainly completely distributive, and so there is a plenary set consisting of regular subgroups which are $Q$-closed. Let $M$ be a $Q$-closed $Q$-value of $g ; M$ is also $P$-closed, and this implies some $a^{m x} \notin M$. $Q$ is very essential over $P$ and thus all the remaining $a^{n x}$ must be in $M$. However, $a^{(m+1) x} \gg a^{m x}$, and hence $a^{(m+1) x} \notin M$, a contradiction.

Case II. There is an $x \in G$ such that $b>a^{x}$ (rel. $P$ ). By definition, replacing $x$ by $-x$, we get that $b \wedge_{P} b^{n(-x)}=0$ or $b=b^{n(-x)}$ for each positive integer $n$; this is equivalent to saying that, for each positive integer $n, b \wedge_{P} b^{n x}=0$ or $b=b^{n x}$. But $b \wedge_{Q} b^{x}>0$ (rel. $Q$ ), since $b>a^{x}$ and $b^{x} \gg a^{x}$ (rel. $Q$ ); once again by Theorem 1.4 in [7] we have $b \gg b^{x}$ or $b^{x} \gg b$ (rel. $Q$ ). Let us suppose the latter holds; we then have the long sequence

$$
b \ll b^{x} \ll \cdots \ll b^{n x} \ll \cdots \quad(\text { rel. } Q) .
$$

Therefore $b \wedge_{P} b^{n x}=0$ for all $n=1,2, \ldots$, and it follows the $b^{n x}$ are pairwise $P$-disjoint. We conclude then that $h=b \vee_{P} b^{x} \vee_{P} b^{2 x} \vee_{P} \cdots$ exists, and as in Case I this leads to a contradiction. An identical argument can be given if $b \gg b^{x}$ (rel. $Q$ ).

The assumption that $Q$ is a proper very $c$-essential extension of $P$ must be false; that is, $P=Q$. Summarizing then

3.2 THEOREM. Let $(G, P)$ be a laterally complete l-group satisfying property (S) and the local representation property. Then $P$ has no proper very $c$-essential extensions.

3.2.1 Corollary. If $H$ and $K$ are o-groups then the cone of $H$ wr $K$ has no proper very c-essential extensions.

3.2.2 COROLlaRY. Let $(G, P)$ be an l-group with a finite basis which also satisfies property (S). Then $P$ has no proper very essential extensions. 
We offer the following item as an interesting sidelight:

3.3 Proposition. Let $(G, P)$ be a laterally complete l-group satisfying property (S) and the local representation property. Either $P$ is a total order, or else $G$ admits no total orders.

Proof. Suppose $P$ is not a total order, and $Q$ is a total order on $G$. We can choose $P$-special elements $a$ and $b$ which are comparable; then, for each $n=1,2$, $\ldots, a \wedge_{P} a^{n x}=0$ or else $a=a^{n x}$.

Case I. $a \wedge_{P} a^{n x}=0$ for all $n=1,2, \ldots$ Let $g=\bigvee_{-\infty}^{+\infty} a^{(2 n-1) x}$ and $h=\bigvee \underbrace{+\infty}_{-\infty} a^{2 n x}$, where $a^{0 x}=a$ and both joins are taken relative to $P$. Then $g$ and $h$ are $P$-disjoint and $(g-h)^{x}=g^{x}-h^{x}=h-g$. However, in view of the fact that $Q$ is a total order, either $g-h$ or $h-g$ must be in $Q$; but by the above, if one is in $Q$ so is the other. This is naturally absurd.

Case II. $a=a^{n x}$ for some positive integer $n$. Let $m$ be the smallest positive integer for which $a=a^{m x}$. Clearly $m>1$, otherwise $a$ would be comparable to $b$. Without loss of generality we may assume $a<a^{x}$ (rel. $Q$ ). Then

$$
a<a^{x}<a^{2 x}<\cdots<a^{m x}=a,
$$

a contradiction.

We must conclude that $G$ admits no total orders.

We close this section with a result which generalizes Corollary 3.2.2.

3.4 TheOREM. Let $(G, P)$ be an l-group satisfying properties $(\mathrm{F})$ and $(\mathrm{S})$. Then $P$ has no proper very essential extensions.

Proof. Suppose $Q$ is a proper very essential extension of $P$. Then there exist $P$-disjoint elements $x$ and $y$ such that $x \gg y$ (rel. $Q$ ); in fact, since $(G, P)$ is finite valued, we may assume $x$ and $y$ are $P$-special. By property (S) there must be a $g \in G$ for which $x^{g}$ and $y$ are comparable.

Case I. $x^{g} \leqq y$ (rel. $P$ ); then $x^{g} \ll x$ (rel. $Q$ ). By the second condition in property (S) we must have $x \wedge_{P} x^{n g}=0$ for all $n=1,2, \ldots$ It follows that the $x^{n g}$ are pairwise $P$-disjoint.

Consider now $x$ and $g$; $x$ cannot be $P$-disjoint to $g$ since $x \wedge_{P} x^{g}=0$. This means the $P$-value of $x$ must be comparable to some $P$-value of $g$; in fact, one can show as in the proof of 1.10 in [7], that $g \gg x$ (rel. $P$ ) and hence $g \gg x^{n g}$ (rel. $P$ ), for each $n=1,2, \ldots$ This contradicts property $(\mathrm{F})$.

Case II. $x^{g}>y$ (rel. $P$ ); then $x \wedge_{Q} x^{g}>0$ (rel. $Q$ ), and so $x^{g} \ll g$ (rel. $Q$ ). Just as in Case I we can show the $x^{n g}$ are pairwise $P$-disjoint and of course each $x^{n g} \ll g$ (rel. $P$ ), once again contradicting property $(\mathrm{F})$.

We therefore conclude that $P$ has no proper very essential extensions, thus proving the theorem.

In view of the results in this section it would be interesting to know just how close property (S) comes to characterizing those l-groups whose cones have no 
proper very essential extensions. Of course, the other assumptions in these theorems were used rather crucially, but it might be possible to refine the proofs in order to show property (S) is a sufficient condition for the nonadmittance of proper very essential extensions. It is far more likely, however, that the definition of property (S) needs to be extended in such a way as to be applicable in more general situations. Consider for example the following "definition" of property (S): for any two incomparable regular subgroups $C$ and $D$ there is an element $g \in G$ such that $-g+C+g \subseteq D$ or vice versa. Moreover, for any such $g$ and each $n=1,2, \ldots, C$ is either incomparable, or equal to $-n g+C+n g$.

In any case, it seems that the existence of very essential extensions is strongly dependent on the amount of "orthogonal translation" by conjugation.

\section{BIBLIOGRAPHY}

1. R. D. Byrd and J. T. Lloyd, Closed subgroups and complete distributivity in lattice-ordered groups, Math. Z. 101 (1967), 123-130. MR 36 \#1371.

2. R. D. Byrd, Complete distributivity in lattice-ordered groups, Pacific J. Math. 20 (1967), 423-432. MR 34 \#7680.

3. P. Conrad, Some structure theorems for lattice-ordered groups, Trans. Amer. Math. Soc. 99 (1961), 212-240. MR 22 \#12143.

4. - The lattice of all convex l-subgroups of a lattice-ordered group, Czechoslovak Math. J. 15 (90) (1965), 101-123. MR 30 \#3926.

5. - A characterization of lattice-ordered groups by their convex l-subgroups, J. Austral. Math. Soc. 7 (1967), 145-159. MR 35 \#5371.

6. __ Introduction à la théorie des groupes réticulés, Université de Paris, Faculté des Sciences de Paris, Secrétariat mathématique, Paris, 1967. MR 37 \#1289.

7. J. Martinez, Essential extensions of partial orders on groups, Trans. Amer. Math. Soc. 162 (1971), 35-61.

8. S. Wolfenstein, Valeurs normales dans un groupe réticulé, Atti Accad. Naz. Lincei Rend. Cl. Sci. Fis. Mat. Natur. (8) 44 (1968), 337-342. MR 38 \#3201.

Department of Mathematics, University of Florida, Gainesville, Florida 32601 